\title{
The soil seed bank buffers long-term compositional changes in annual plant communities
}

\author{
Niv De-Malach ${ }^{1}$, Marcelo Sternberg ${ }^{1}$, and Jaime Kigel ${ }^{2}$ \\ ${ }^{1}$ Tel Aviv University \\ ${ }^{2}$ Hebrew University
}

May 11, 2020

\begin{abstract}
Ecological theory predicts that the soil seed bank stabilizes the composition of plant communities in the face of environmental variability. Using one of the longest seed bank-vegetation databases, we tested whether the composition of the seed bank is more stable than the standing vegetation in annual communities across a rainfall gradient. The composition of the seed bank differed from the vegetation throughout the years with a higher abundance of small-seeded and persistent-seeded species. Year-to-year variability in composition increased with increasing aridity, but its magnitude was similar in the seed bank and the vegetation. Importantly, the rate of long-term compositional change was much slower in the seed bank. These results support the hypothesis that the seed bank can buffer against climatic shifts and increases the resistance of plant communities to directional trends. We conclude that the seed bank plays a crucial role in the stability of plant communities under global changes.
\end{abstract}

\section{Hosted file}

seed bank stabilitiy Ecology letters.pdf available at https://authorea.com/users/320586/ articles/450111-the-soil-seed-bank-buffers-long-term-compositional-changes-in-annualplant-communities 